\title{
Evaluation of Movement of Patients with Parkinson's Disease Using Wearable MoCap System and Bilateral Cyclograms
}

\author{
Slavka Viteckova, Patrik Kutilek, Radim \\ Krupicka, Barbora Adamova, Zoltan Szabo, \\ Ana Carolina DAngeles Mendes de Brito \\ Faculty of Biomedical Engineering \\ Czech Technical University in Prague \\ Kladno, Czech Republic \\ viteckova@fbmi.cvut.cz, kutilek@fbmi.cvut.cz, \\ krupicka@fbmi.cvut.cz, szabo@fbmi.cvut.cz,
}

\begin{abstract}
The article focuses on a method of quantifying movement of patients with Parkinson's disease (PD) using wearable motion capture (MoCap) system and bilateral cyclograms. The method uses cyclogram characteristics such as the slope, second moment of area and the area of the cyclogram to compare movements of left and right side of body. For symmetric movement, cyclogram should lie on a symmetry line passing through the origin inclined at an angle $45^{\circ}$. To study the body movement, measurement was performed using accelerometers (Xsens Technologies B.V.). Four accelerometers were placed on subject's arms and legs to measure accelerations in the anterior-posterior, medial-lateral and inferior-superior directions. Subjects (patients and healthy subjects) accomplished an extended Timed Up \& Go Test (TUG). The data from the accelerometers were preprocessed, and then the bilateral cyclograms of two synchronized accelerations were created and evaluated. Using the cyclograms we confirmed the hypothesis that patients with PD have asymmetrical movements of the limbs. Omitting medication 24 hours before the measurement in patients with early stage PD had little effect on the synkinesias of gait. Findings suggest the possibility of the designed application of wearable MoCap system and bilateral cyclograms for use in drug therapy by evaluating postural stability via measuring accelerations. Thus, method using accelerometers and bilateral cyclograms is newly proposed for patients with PD.
\end{abstract}

Keywords - accelerometer; bilateral cyclogram; gait; symmetry; Parkinson's disease

\section{INTRODUCTION}

Gait disturbance in patients with Parkinson's disease (PD) is a consequence of bradykinesia and postural instability. PD leads to a reduction in the movement of the upper limbs, freezing of gait, slow gait, etc. $[1,2]$. In the case of rigidity (muscle stiffness), the muscles of limbs are stretched at rest, which put increased resistance during any movement. Rigidity in patients suffering PD is manifested as e.g. missing swinging the upper limbs when walking, $[1,2$, 3]. Moreover, PD leads to disturbances in the synkinesia. The most important indicator (parameter) of synkinesia of healthy gait is symmetry of movement, [2, 4]. One of the signs of $\mathrm{PD}$ is

\author{
barbora.adamova@fbmi.cvut.cz, ana- \\ Jana Kopecka \\ 1st Faculty of Medicine \\ Charles University \\ Prague, Czech Republic \\ jana.kopecka@vfn.cz
} carolina.dangeles-mendes-de-brito@fbmi.cvut.cz

asymmetric movement of the upper limbs in terms of the reduction, [2].

Symmetry is still primarily evaluated using a variety of statistical methods and coefficients $[5,6]$. Unfortunately, these methods do not allow to analyze the entire cycles of gait but only kinematic motion data at specific time $[2,7]$.

In our work we focus on the study of impact of Parkinson's disease on synkinesias (i.e. symmetry of movement) during walking. Article focuses on the calculation of symmetry of limb movement using accelerometer data recorded by Xsens system (Xsens Technologies B.V.). Method for evaluating the symmetry is based on cyclograms because the method allows to analyze symmetry of entire cycles of gait, $[8$, 9]. For evaluation of the symmetry of the movement are used the so-called bilateral cyclograms, $[10,11]$. In the past the bilateral cyclograms were used only for evaluation angular movements, i.e. joint angles. Thus, diagrams are called bilateral angle-angle diagrams, $[10,11]$. In our case, we will use bilateral cyclograms for evaluating accelerations of upper and lower limb movements during gait. Bilateral cyclograms in conjunction with accelerometer data has not been used before for evaluating the movement. New way of application of accelerometer data and bilateral cyclograms can provide new clinical use in the treatment of patients with PD.

\section{MethodS AND MATERIALS}

\section{A. Participants}

Kinematic data were recorded from 19 patients with PD (including 13 males and 6 females) with mild to moderate PD, mean age 68.9 (SD 7.1) years and 14 age matching healthy adult subjects (including 6 males and 8 females), mean age 65 (SD 8.2) years without history of neuropsychiatric disorders and without any gait impairment. Every PD patient was tested in his $\mathrm{ON}$ and OFF state. The OFF state was achieved more than 12 hours after withdrawal of anti-Parkinson medication while the ON state was 1.5 hours after taking the usual medication again. 


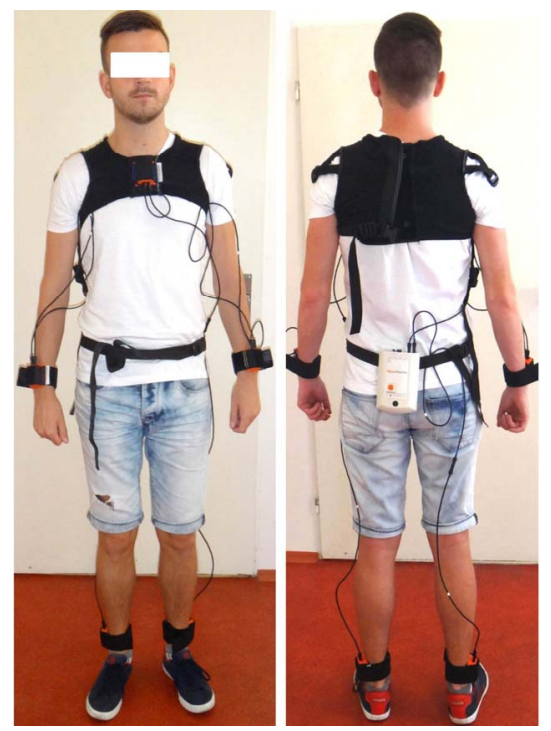

Figure 1. Arrangement of the Xsens system with five three-axis IMU used to measure accelerations of body segments, [2].

\section{B. Test Procedure and Measurement Equipment}

Xbus Master (Xsens Technologies B.V.), lightweight $(330 \mathrm{~g})$ and portable device using MTx units for orientation and acceleration measurement of body segments, was used for measurement of segment movements. MTx unit with an embedded accelerometer and gyroscope is accurate IMU measuring drift-free 3-D orientation and 3-D acceleration. Kinematic data were recorded from 5 gyro-accelerometers with data sampling rate of 100 $\mathrm{Hz}$. Four accelerometers were attached on the patient's arms and legs to measure magnitudes of accelerations in medial-lateral (x), anterior-posterior $(\mathrm{y})$ and inferior-superior ( $\mathrm{z}$ ) directions. Accelerometer units were symmetrically attached on the lateral shank of each lower leg, $4 \mathrm{~cm}$ above the ankle joint, on the dorsum of each wrist and the chest, $2 \mathrm{~cm}$ below the sternal notch, see Fig. 1.

Subjects accomplished an extended Timed Up \& Go Test (TUG). Each subject was observed and timed while he rises from an arm chair during TUG, walks 7 meters, turns, walks back, and sits down again. Two repeated collections of TUG were recorded for each subject (i.e. patients and healthy subjects). One of two TUG trial's accomplishment was randomly selected and processed.

\section{Method of Data Processing}

The raw data were real-time stored to the computer via original manufacturer's software Xsens MT Manager. All preprocessing and analysis were carried out offline on MacBook Pro using the MatLab (MatLab R2010b, Mathworks, Inc., Natick, MA, USA) programming environment. Before further processing, the raw acceleration signal was low-pass filtered with zero-phase 3th order Butterworth filter with a $4 \mathrm{~Hz}$ corner frequency. The TUG subcomponents, namely sit-to-stand, gait, turning, and turn-to-sit, were automatically identified $[2,12]$. The acceleration of gait component between sit-to-stand and turn component was extracted and used for further analysis.

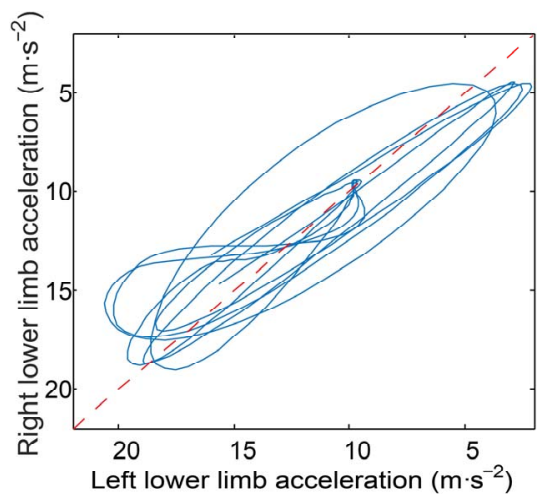

Figure 2. Example of bilateral cyclogram of synchronized accelerations of left and right lower limb.

We employed the method of cyclograms to assess symmetry of lower and upper limbs movement during gait. For a symmetric gait, two acceleration time-series (trajectories) from contralateral limbs should be identical and a bilateral cyclogram should lie on a symmetry line. The symmetry line is a straight line passing through the origin inclined at an angle 45 degrees, see Fig. 2. We can also compute the area within the cyclogram (in the case of acceleration, $\mathrm{m}^{2} \cdot \mathrm{s}^{-}$ 4 is unit) and its orientation to evaluate the rate of asymmetry, [4, 11]. The cyclograms may consist of self-intersecting loops or they have a complex shape. Although the cyclogram may be complex and asymmetrical, its surface may be very small. For example, a crescent shape can leads to a small area, although it is an asymmetrical gait, see, [4]. For this reason, we must also use the second moment of area (in the case of acceleration, $\mathrm{m}^{4} \cdot \mathrm{s}^{-8}$ is unit) to describe the shape of the cyclogeam. Calculation of characteristics of the cyclogram is described in detail in $[4,10,11]$.

We can express mathematically cyclogram deviation from the cyclogram of ideal symmetric gait to obtain a quantification of asymmetry, see [11]. The triplet of geometric properties of cyclograms, namely the area within the cyclogram $(S)$, angle between the symmetry line and the principal axis of the cyclogram, $(\alpha)$, and minimum second moment of cyclogram area, (J), can be represented by point in 3-D space, Fig.3. The ideal point for symmetric gait has coordinates $(0$, $45,0),[11]$. By using only those three characteristics together, significant asymmetry can be identified. Regarding identification of asymmetry using quantitative parameters of the cyclogram, only two

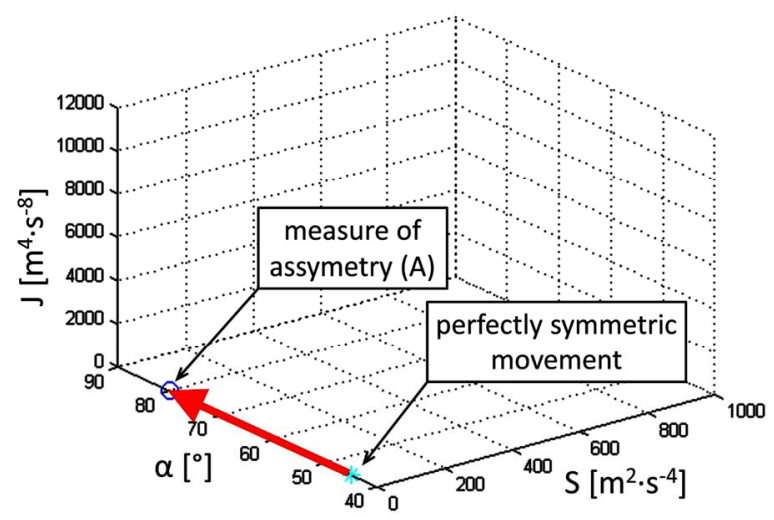

Figure 3. Example of three symmetry quantifiers plotted for body segments (ankle joints) accelerations of $\mathrm{ON}$ patient during gait. 
parameters do not allow to identify the significant asymmetry. During asymmetric walking can be situations when the use of only two parameters (e.g. area of cyclogram and second moment of cyclogram area) can show absolute symmetry. However, this can lead to misleading quantitative results. Only the use of all three parameters can safely detect and quantify the significant asymmetry, [4, 10, 11]. For quantitative evaluation of all three cyclogram characteristics, only one symmetry indicator was used, [11]. The point in $3 \mathrm{D}$ space of geometric properties of cyclogram was determined for each measurement, see Fig.3. Then, the asymmetry, $A$, was defined as distance from the measurement point and ideal point, see Fig. 3, [11]:

$$
A=\sqrt{\left(\alpha_{M}-\alpha_{I}\right)^{2}+\left(S_{M}-S_{I}\right)^{2}+\left(J_{M}-J_{I}\right)^{2}} .
$$

The symmetry indicator based of cyclogram characteristics was calculated for the measured movement of upper and lower limbs of all patients and healthy subjects.

\section{Statistical Analysis}

Statistical analyses were performed to examine whether a method of cyclograms is able to distinguish healthy subject from PD patients. Shapiro-Wilk test was used to verify the normality of parameters in each observed dataset. The assumption of normal data distribution in the observed datasets has been rejected (significance level $\mathrm{p}=0.05$ ). Therefore, nonparametric Wilcoxon signed rank test was used to compare statistical significance differences in movement symmetry between PD patients in ON and OFF state. Nonparametric Wilcoxon rank sum test was used to compare PD patient data to norms data. The significance level was set to $p<0.05$, resp. $p<0.01$.

\section{RESULTS}

\section{A. Lower Limbs Symmetry}

Using statistical analysis of TUG test based on calculated values of cyclogram properties of lower limbs data before turning we revealed significant difference between reference group and PD patients without medication in $\mathrm{x}$ and $\mathrm{z}$ direction. The difference between symmetry value is also significant for PD patients with and without medication in $\mathrm{Z}$ direction (see Table I.). In other cases, the difference in symmetry value is not statistically significant.

\section{B. Upper Limbs Symmetry}

In the case upper limbs symmetry, we could see the statistically significant differences between reference group and PD patients with medication in $\mathrm{x}$ direction. There is also statistically significant difference between PD patients with medication and $\mathrm{PD}$ patients without medication in $\mathrm{x}$ direction (see Table II.). In other cases, the difference in symmetry value is not statistically significant.

\section{DISCUSSION}

We tested and verified new method that is derived from the geometric properties of angle-angle
TABLE I. STATISTICAL EVALUATION OF UPPER LiMBS Cyclogram Symmetry Value Between Tested SubJeCts GROUPS (P-VALUE).

\begin{tabular}{|c|c|c|c|}
\hline Dir & ON/OFF & N/ON & N/OFF \\
\hline $\mathbf{x}$ & $<0.01^{*}$ & $<0.01^{*}$ & 0.33 \\
\hline $\mathbf{y}$ & 0.50 & 0.81 & 0.87 \\
\hline $\mathbf{z}$ & 0.28 & 0.39 & 0.07 \\
\hline \multicolumn{3}{|c|}{$\begin{array}{c}\text { Dir-direction of acceleration, ON-patients with } \\
\text { medication, OFF-patients without medication, N- } \\
\text { norms, *-significant difference. }\end{array}$} \\
\hline
\end{tabular}

TABLE II. STATISTICAL EVALUATION OF LOWER LIMBS CyClogram SyMmetry VALUE BETWEen TESTED SuBJECTS GROUPS (P-VALUE).

\begin{tabular}{|c|c|c|c|}
\hline Dir & ON/OFF & N/ON & N/OFF \\
\hline $\mathbf{x}$ & 0.08 & 0.13 & $<0.01^{*}$ \\
\hline $\mathbf{y}$ & 0.18 & 0.30 & 0.13 \\
\hline $\mathbf{z}$ & $<0.01^{*}$ & 0.73 & $<0.01^{*}$ \\
\hline \multicolumn{2}{|c|}{$\begin{array}{c}\text { Dir-direction of acceleration, ON-patients with } \\
\text { medication, OFF-patients without medication, N- } \\
\text { norms, *-significant difference. }\end{array}$} \\
\hline
\end{tabular}

diagrams, $[10,11]$. To our knowledge, this is the first report of the use of symmetry quantification technique based on the symmetry indicator determined from the characteristics of acceleration-acceleration diagram. After obtaining the acceleration of the upper and lower limb of subjects during TUG test, we created the bilateral cyclograms in MatLab software. The acellerastion-acceleration diagrams were created for patients with and without medication and healthy subjects. We obtained cyclograms characteristics (i.e. area of cyclogram, angle between the symmetry line and the principal axis of the cyclogram and minimum second moment of cyclogram area) and then the symmetry indicator calculated for all subjects, [4].

The main finding is that the medication had little effect on the gait symmetry of patients with PD. Omitting medication 24 hours before the measurement in patients with early stage PD had little effect on the synkinesias of gait. The results are consistent with previous studies of $\mathrm{PD},[2]$. This result confirms the fact that antiparkinson medication only suppresses the symptoms and delay the other symptoms of the disease but does not cure the disease, [2]. Grajic et al. [13] concluded that symmetry in de novo drug naive PD patients remains preserved, [2].

Comparing the movement of healthy subjects and patients, reduced symmetry between both arms has been reported in PD, [14, 15]. Results obtained using the cyclograms are in line with our previous studies, [2]. The reduced symmetry of entire arm swing wave during PD gait is confirmed in this study. Also, calculated parameters of cyclograms show that the arms' synkinesia is not well regulated in PD. This supports the hypothesis that PD negatively affects locomotor central pattern generation and basal ganglia regulation, see $[2,7]$.

Since the gait symmetry of patients with PD is assessed using the spatiotemporal gait cycle parameters (i.e. step length, step time, the variability of steps and walking speed) in previous works, so we chose calculation of the cyclogram characteristics of right and left limb movements to study the synkinesia, $[4,10,11]$. In our case, synkinesia is not determined 
from the basics parameters of the gait cycle at specific time, the cyclogram describing entire cycles of gait was used. The method using bilateral accelerationacceleration diagram for study synkinesia of patients with Parkinson's disease has never been used before. In the past, methods using bilateral cyclograms were only used to study gait of patients after a stroke or amputations. The advantage of the method is the analysis of the entire gait cycle unlike the calculation of the coefficients, which are mainly used for the quantification of gait symmetry at specific time. A limitation on the calculation of coefficients is a relatively low sensitivity and inability to determine the place where asymmetry arise $[6,15]$. The other advantage of used method is the uniform approach to gait symmetry in all movement direction. And thus, the possible comparison of the impact of Parkinson's disease on movement in different directions can be applied, [2].

There are limitations to our study. The main weakness of this study is age of subjects. The sample of the subjects was not representative of the larger population, [2]. However, to test the basic attributes of the method proposed for the study of postural stability in this preliminary study, a sample of older patients and older healthy subjects is sufficient (i.e. with similar age as patients), just as it was in similar works focused on the postural stability evaluation, [17]. In the future, it would be advisable to do research, which would bring together previously untreated patients, patients using the medicament and the reference group, [2]. Also, according to the [7], it is suitable use more sensors (3 for each limb, chest and back) for the measurement which could contain more information for calculation of symmetry, [2].

\section{CONCLUSION}

Method using bilateral cyclograms is newly proposed for evaluation of the limb accelerations and tested on patients with PD. The symmetry indicator based on three cyclogram goniometry characteristics can be used to study of the complex movements and induces the deviations of the cyclogram shape from the ideal one. Using the symmetry indicator of cyclogram, we confirmed the hypothesis that patients with PD have asymmetrical movements of the limbs. This new method also points to the movement coordination of the upper and lower extremity of healthy subjects and patients. We can designate the method using bilateral cyclogram (also called bilateral acceleration-acceleration diagram) as method which is suitable for evaluation of gait symmetry and synkinesis in practice.

\section{ACKNOWLEDGMENT}

This work was done in the FBE CTU in Prague in the framework of research project SGS SGS16/109/OHK4/1T/17 and AZV Grant no. 1628119a "Analysis of movement disorders for the study of extrapyramidal diseases mechanism using motion capture camera systems".

\section{REFERENCES}

[1] M. Bares, "Diagnostika a klinické př́znaky Parkinsonovy nemoci," Neurologie pro praxi, vol. 2, no 1, pp. 22-24, 2001.

[2] S. Viteckova, P. Kutilek, J. Lenartova, J. Kopecka, D. Mullerova and R. Krupicka, "Evaluation of movement of patients with Parkinson's disease using accelerometers and method based on eigenvectors," Proc. of 17th International Conference on Mechatronics, Prague, Czech Republic, 2016.

[3] J. Benetin, F. Cibulcik, P. Turcani and P. Valkovic, Ako žit's Parkinsonovou chorobou, Bratislava: Lundbeck Slovensko, 2007.

[4] P. Kutilek, S. Viteckova, Z. Svoboda, V. Socha and P. Smrcka, "Kinematic quantification of gait asymmetry based on characteristics of angle-angle diagrams," Acta. Polytech. Hung., vol. 11, no. 5, pp. 25-38, 2014.

[5] S. J. Crenshaw and J. G.Richards, "A method for analyzing joint symmetry and normalcy, with an application to analyzing gait," Gait. Posture., vol. 24, no. 4, pp. 515-521, 2006.

[6] M. Błażkiewicz, I Wiszomirska and A. Wit, "Comparison of four methods of calculating the symmetry of spatial-temporal parameters of gait," Acta. Bioeng. Biomech., vol. 16, no. 1, 2014, pp. 29-35.

[7] X. Huang, J. M. Mahoney and M. M. Lewis, "Both coordination and symmetry of arm swing are reduced in Parkinson's disease," Gait. Posture., vol. 35, no. 3, pp. 373377, 2012.

[8] O. Hajny and B. Farkasova, "A study of gait and posture with the use of cyclograms," Acta Polytechnica, vol. 50, no. 4, pp. 48-51, 2010.

[9] P. Kutilek and B. Farkasova, "Prediction of lower extremities' movement by angle-angle diagrams and neural networks," Acta. Bioeng. Biomech., vol.13, no. 2, pp. 57-65, 2011.

[10] P. Kutilek, S. Viteckova, Z. Svoboda and P. Smrcka, "Kinematic quantification of gait asymmetry in patients with peroneal nerve palsy based on bilateral cyclograms," J. Musculoskelet. Neuronal. Interact., vol. 13, no. 2, pp. 244$250,2013$.

[11] A. Goswami, "Kinematics quantification of gait symmetry based on bilateral cyclograms," Proc. of the XIXth Congr. of the Int. Society of Biomechanics, Dunedin, New Zealand, 2003.

[12] A. Salarian, F. Horak, C. Zampieri, P. Carlson-Kuhta, J. Nutt and K. Aminian, " iTUG, a Sensitive and Reliable Measure of Mobility,” IEEE Trans. Neural. Syst. Rehabil. Eng., vol. 8, no. 3, pp. 303-310, 2010.

[13] M. Grajic, I. Stankovic, S. Radovanovic and V. Kostic, "Gait in drug naïve patients with de novo Parkinson's disease altered but symmetric," Neurol. Res., vol. 37, no. 8, pp. 712716,2015

[14] C. Zampieri, A. Salarian, P. Carlson-Kuhta, K. Aminian, J. G. Nutt and F. G. Horak, "An instrumented timed up and go test characterizes gait and postural transitions in untreated Parkinson's disease," J. Neurol. Neurosurg. Psychiatry., vol. 81, no.2, pp.171-176, 2010.

[15] M. D. Lewek, R. Poole, J. Johnson, O. Halawa and X. Huang, "Arm swing magnitude and asymmetry during gait in the early stages of Parkinson's disease," Gait. Posture., vol. 31, no. 2, pp.256-260, 2010.

[16] R. Seliktar and J. Mizrahi, "Some gait characteristics of below-knee amputees and their reflection on the ground reaction forces," Eng. Med., vol. 15, no. 1, pp. 27-34, 1986.

[17] D. Pan, R. Dhall, A. Lieberman and D. Petitti, "A mobile cloud-based Parkinson's disease assessment system for homebased monitoring," JMIR Mhealth Uhealth, vol. 3, no. 1, pp.e29, 2015. 